\title{
Kontos, Maria: Die desintegrativen Folgen des öffentlichen Integrationsdiskurses - Eine biographieanalytische Untersuchung mit Migrantinnen und Migranten.
}

\author{
Leverkusen: Barbara Budrich 2020. 231 Seiten. Preis: $€ 19,99$ \\ (e-book)
}

\author{
Sabrina Schmidt \\ Angenommen: 6. März 2021 / Online publiziert: 24. März 2021 \\ (C) Der/die Autor(en) 2021
}

Die kommunikative Aushandlung kollektiver Identitäts-, Zugehörigkeits-, und Teilhabefragen gehört zu den Kernprozessen postmigrantischer Gesellschaften. Aus der Perspektive kritischer Öffentlichkeitstheorien bemisst sich deren Qualität an der Frage, inwiefern gesellschaftliche Ungleichheitsstrukturen und hegemoniale OtheringDiskurse bestimmte Gesellschaftsgruppen von der Mitsprache ausschließen. Die empirische Kommunikationswissenschaft richtet ihren Blick hier klassischerweise auf Repräsentationsproblematiken, wobei die lebensweltliche Verarbeitung medienöffentlicher Debatten um Migration und Integration - insbesondere durch Personen mit Migrationsgeschichte, die von ihnen direkt betroffen sind - insgesamt noch wenig ausgeleuchtet ist. Maria Kontos hat diese Lücke erkannt und fragt mit ihrer Studie nach den sozialen, emotionalen und kognitiven Auswirkungen, die öffentliche Integrationsdiskurse auf die Selbstwahrnehmung und gesellschaftliche Positionierung von MigrantInnen haben.

Im Mittelpunkt ihrer empirischen Studie stehen ausgewählte rekonstruktive Fallanalysen aus einem Sample von insgesamt 48 Interviews mit MigrantInnen aus früheren Anwerbeländern sowie nachfolgenden Generationen. Kontos hat diese mittels biographisch-narrativer Interviews befragt, wobei diese Methodenwahl plausibel erscheint, geht es ihr doch vor allem um lebensweltliche Relevanzstrukturen und Wandlungen sowie Kontinuitäten in den Selbstbetrachtungen der MigrantInnen. Mit direktem Bezug zur Sarrazin-Debatte der Jahre 2009-2010 vermutet die Autorin dabei grundlegend, ,dass der öffentliche Integrationsdiskurs, der negative Bilder des Selbst transportiert, als symbolischer und materiell-rechtlicher Ausschluss und als Missachtung erfahren werden könnte und dass diese Erfahrungen zu Reparaturreak-

Dr. des. S. Schmidt $(\bowtie)$

Erlanger Zentrum für Islam und Recht in Europa EZIRE, Friedrich-Alexander-Universität

Erlangen-Nürnberg, Bohlenplatz 6, 91054 Erlangen, Deutschland

E-Mail: sabrina.b.schmidt@fau.de 
tionen und damit zu einer Veränderung der sozialen Positionierung führen dürfte“ (S. 19). Kontos geht es darum, die (destruktiven) Folgeerscheinungen herauszuarbeiten, die sich aus den rassistischen Einlassungen Sarrazins gegenüber MuslimInnen und MigrantInnen ergeben. Dabei wird ihre Analyse zeigen, dass diese nicht selten durch sozialkritische und selbst-empowernde Reaktionen der Interviewten erfolgreich gekontert werden.

Auch wenn sich der zentrale Erkenntniswert der Arbeit zweifelsohne aus dem Datenmaterial und aus dessen konzeptgeleiteter Analyse speist, beinhalten die vielfältigen theoretischen Grundlegungen bereits einige gelungene Synthesen. Dabei startet Kontos zunächst eher konservativ mit einer Rekonstruktion von Hartmut Essers Mainstreammodell von Integration, das sie jedoch kritisch diskutiert und alternativen Konzepten der neueren Migrationsforschung gegenüberstellt. Anschließend leitet sie zügig über in diskurstheoretische Ausführungen, die es ihr erlauben, das Verhältnis von Diskurs, Subjekt und Biographie umfassend zu erörtern. Unter Rückgriff auf wissenssoziologische Überlegungen von Alfred Schütz, Stuart Halls Artikulationskonzept sowie den auf Jacques Lacan zurückgehenden Begriff des Phantasmas verweist Kontos nicht nur auf die Produktivität der RezipientInnen im Umgang mit Mediendiskursen, sondern legt auch ein besonderes Augenmerk auf die latenten und unausgesprochenen Elemente in öffentlichen Debatten über Migration.

Grundsätzlich gelungen ist zudem die Verzahnung des Theoriefundaments mit aktuellen Erkenntnissen aus der empirischen Integrationsforschung und Kommunikationswissenschaft, auch wenn man sich hier eine stärkere Berücksichtigung medienwissenschaftlicher Arbeiten aus dem Feld der Aneignungsforschung gewünscht hätte. Dies wäre auch deswegen naheliegend gewesen, weil die Arbeit in ihrer theoretischen Anlage weniger von direkten Medienwirkungen, denn von dynamischen Interaktionen zwischen Medientext und RezipientInnen ausgeht.

Im daran anschließenden Ergebnisteil zeichnet Kontos „,ein differenziertes Bild von Integrationsdiskurswahrnehmung und -verarbeitung“ (S. 63), wobei sie zunächst zwei Globaltypen rekonstruiert: InterviewpartnerInnen, die den Integrationsdiskurs als persönlich irrelevant betrachten und jene, die die Anrufung des Diskurses annehmen und sich als dessen Angriffsziel erkennen. Kontos fokussiert im weiteren Verlauf auf den zweiten Typus, wobei es der Autorin auf eindrückliche Weise gelingt, die vielfältigen lebensweltlichen Verarbeitungsweisen, Reflexionsmuster und biographischen Handlungsanschlüsse der InterviewpartnerInnen herauszuarbeiten. Einführenden Kurzporträts folgen dichte Beschreibungen der Haupterzählungen, die sich zunächst auf die persönliche Migrationsgeschichte fokussieren. Im Anschluss erörtert Kontos die argumentativen, affektiven, alltagskommunikativen und biographischen Einordnungen der Sarrazin-Debatte durch die Interviewten. Dank einer sorgfältigen theoretischen Vorbereitung ist es der Autorin hier möglich, das Interviewmaterial strukturiert aufzubereiten, Analyseerkenntnisse dicht am Material zu formulieren und die ausgewählten Fälle punktuell miteinander zu vergleichen.

Auch wenn es sich bei dieser Studie um keine klassische kommunikationswissenschaftliche Arbeit handelt, sind die theoretischen und methodologischen Vorbetrachtungen sowie vor allem die empirischen Einblicke in die lebensweltlichen Aneignungsmuster migrantischer Personen für Medien- und KommunikationswissenschaftlerInnen von erheblichem Erkenntniswert. Einerseits füllt sie (in Ansätzen) die 
im öffentlichen Diskurs klaffende Repräsentationslücke, die MigrantInnen nur selten selbst zu Wort kommen lässt. Zum anderen macht sie die Kontinuität verkennender Integrationsdebatten deutlich, die sich nicht auf Sarrazins Thesen beschränken, und die derzeit in antimigrantischer Hate Speech im Internet ihren drastischen Ausdruck finden.

Funding Open Access funding enabled and organized by Projekt DEAL.

Open Access Dieser Artikel wird unter der Creative Commons Namensnennung 4.0 International Lizenz veröffentlicht, welche die Nutzung, Vervielfältigung, Bearbeitung, Verbreitung und Wiedergabe in jeglichem Medium und Format erlaubt, sofern Sie den/die ursprünglichen Autor(en) und die Quelle ordnungsgemäß nennen, einen Link zur Creative Commons Lizenz beifügen und angeben, ob Änderungen vorgenommen wurden.

Die in diesem Artikel enthaltenen Bilder und sonstiges Drittmaterial unterliegen ebenfalls der genannten Creative Commons Lizenz, sofern sich aus der Abbildungslegende nichts anderes ergibt. Sofern das betreffende Material nicht unter der genannten Creative Commons Lizenz steht und die betreffende Handlung nicht nach gesetzlichen Vorschriften erlaubt ist, ist für die oben aufgeführten Weiterverwendungen des Materials die Einwilligung des jeweiligen Rechteinhabers einzuholen.

Weitere Details zur Lizenz entnehmen Sie bitte der Lizenzinformation auf http://creativecommons.org/ licenses/by/4.0/deed.de.

Dr. des. Sabrina Schmidt ist wissenschaftliche Mitarbeiterin am Erlanger Zentrum für Islam und Recht in Europa (EZIRE), Friedrich-Alexander-Universität Erlangen-Nürnberg. 